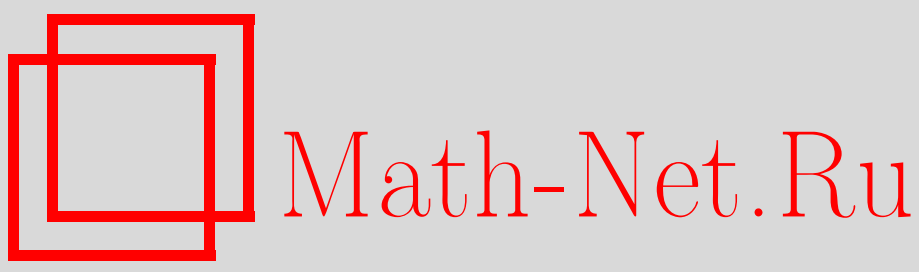

С. Н. Кудрявцев, Приближение оператора частного дифференцирования ограниченными операторами на классе функций конечной гладкости, Матем. сб., 1996, том 187, номер 3, 75-92

DOI: https://doi.org/10.4213/sm117

Использование Общероссийского математического портала Math-Net.Ru подразумевает, что вы прочитали и согласны с пользовательским соглашением

http://www. mathnet.ru/rus/agreement

Параметры загрузки:

IP : 54.209 .52 .79

26 апреля 2023 г., 12:36:37 
УДК 517.5

\author{
С.Н. Кудрявцев
}

\title{
Приближение оператора частного дифференцирования ограниченными операторами на классе функций конечной гладкости
}

\begin{abstract}
В статье установлена слабая асимптотика убьвания соответствующей величины в задаче о наилучшей точности приближения на классе функций с заданной мажорантой модулей непрерьвности старших производных оператора частного дифференцирования ограниченными операторами, действующими из одного пространства с интегральной нормой в другое.

Библиограбоя: 6 названий.
\end{abstract}

\section{Введение}

В работе рассматривается задача о наилучшем приближении по норме пространства $L_{q}\left(I^{d}\right)$ оператора частного дифференцирования $\mathscr{D}^{\lambda}$ ограниченными операторами из $L_{s}\left(I^{d}\right)$ в $L_{q}\left(I^{d}\right)$, норма которых не превосходит $\rho$, на классе $F_{p}^{l, \omega}\left(I^{d}\right)$, где $F_{p}^{l, \omega}\left(I^{d}\right)$ состоит из функций, определенных на единичном кубе $I^{d}$, обобщенные частные производные до порядка $l$ которых принадлежат $L_{p}\left(I^{d}\right)$, а модули непрерывности в $L_{p}\left(I^{d}\right)$ производных порядка $l$ имеют мажоранту $\omega$. Изучается поведение в зависимости от $\rho$ величины наилучшей точности приближения в этой задаче.

Задача о наилучшем приближении на классе $F_{p}^{l, \omega}\left(I^{d}\right)$ оператора дифференцирования $\mathscr{D}^{\lambda}$ ограниченными операторами из $L_{s}\left(I^{d}\right)$ в $L_{q}\left(I^{d}\right)$ является частным случаем задачи, поставленной в [1], о наилучшем приближении на некотором множестве неограниченного линейного оператора ограниченными. Обзор результатов и список литературы по этой тематике содержится в [2]. Из последних работ приведем [3]-[5]. Отметим, что в [3], [4] приближение дифференциального оператора осуществляется на классе соболевского типа функций многих переменных, определенных во всем пространстве, а в [5] - на классе функций, имеющих на кубе непрерывные частные производные до определенного порядка, с заданной мажорантой модулей непрерывности старших производных. Причем оценки наилучшей точности приближения найдены в ситуации, когда приближение производится ограниченными операторами, переводящими пространство, на котором они определены, в себя.

Полученные в настоящей работе оценки точности приближения в описанной выше задаче обобщают один из результатов, установленных в [5]. При этом применяемая ниже техника вывода интересующих нас оценок сушественно отличается от той, которая использована для получения соответствующих соотношений в [3]-[5]. Отметим еще, что подобно тому, как это сделано ниже, автором найдены аналогичные оценки в случае приближения оператора дифференцирования на классе функций Бесова. 
Работа состоит из введения и двух параграфов, в первом из которых проведена оценка сверху изучаемой величины, а во втором - оценка снизу.

\section{§1. Оценка сверху величины наилучшей точности приближения оператора $\mathscr{D}^{\lambda}$ ограниченными операторами из $L_{s}\left(I^{d}\right)$ в $L_{q}\left(I^{d}\right)$ на классе $F_{p}^{l, \omega}\left(I^{d}\right)$}

1.1. В этом пункте введем некоторые определения и обозначения, а также сформулируем доказанные в [6] вспомогательные утверждения, которые потребуются для доказательства основного результата $\S 1$.

Как обьчно, через $\mathbb{Z}_{+}^{d}$ будем обозначать множество

$$
\left\{\lambda=\left(\lambda_{1}, \ldots, \lambda_{d}\right) \in \mathbb{Z}^{d}: \lambda_{j} \geqslant 0, j=1, \ldots, d\right\},
$$

через $\mathbb{Z}_{+l}^{d}, l \in \mathbb{Z}_{+}$, обозначим множество

$$
\left\{\lambda \in \mathbb{Z}_{+}^{d}:|\lambda| \leqslant l\right\}
$$

где $|\lambda|=\sum_{j=1}^{d} \lambda_{j}$, а через $\mathbb{Z}_{+l}^{\prime d}$ обозначим множество

$$
\left\{\lambda \in \mathbb{Z}_{+}^{d}:|\lambda|=l\right\}
$$

При $l \in \mathbb{Z}_{+}, d \in \mathbb{N}$ через $\mathscr{P}_{l, d}$ обозначим пространство вешественных полиномов степени не выше $l$ от $d$ переменных, т.е. пространство всех функций $f: \mathbb{R}^{d} \rightarrow \mathbb{R}$ вида $f(x)=\sum_{\lambda \in \mathbb{Z}_{+l}^{d}} a_{\lambda} x^{\lambda}$, где $x_{\lambda}=x_{1}^{\lambda_{1}} \cdot \ldots \cdot x_{d}^{\lambda_{d}}$.

Для измеримого по Лебегу множества $D \subset \mathbb{R}^{d}$ при $1 \leqslant p \leqslant \infty$ через $L_{p}(D)$, как обычно, обозначается пространство всех вешественных измеримых на $D$ функций $f$, для которых определена норма

$$
\|f\|_{L_{p}(D)}= \begin{cases}\left(\int_{D}|f(x)|^{p} d x\right)^{1 / p}, & 1 \leqslant p<\infty, \\ \underset{x \in D}{\sup _{x \in D}|f(x)|,} & p=\infty .\end{cases}
$$

Для области $D \subset \mathbb{R}^{d}$ при $1 \leqslant p \leqslant \infty, l \in \mathbb{Z}_{+}$через $W_{p}^{l}(D)$ обозначается пространство всех функций $f \in L_{p}(D)$, для которых для каждого $\lambda \in \mathbb{Z}_{+l}^{d}$ обобщенная производная $\mathscr{D}^{\lambda} f=\frac{\mathscr{D}^{|\lambda|} f}{\mathscr{D}^{\lambda_{1}} x_{1} \cdots \mathscr{D}^{\lambda} x_{d}}$ принадлежит $L_{p}(D)$, с нормой

$$
\|f\|_{W_{p}^{l}(D)}=\sum_{\lambda \in \mathbb{Z}_{+l}^{d}}\left\|\mathscr{D}^{\lambda} f\right\|_{L_{p}(D)}
$$

В пространстве $\mathbb{R}^{d}$ фиксируем норму

$$
\|x\|=\max _{j=1, \ldots, d}\left|x_{j}\right|, \quad x \in \mathbb{R}^{d} .
$$

Пусть $\omega$ - модуль непрерывности, т.е. $\omega$ - непрерывная вешественная функция на $[0, \infty)$ такая, что

$$
0=\omega(0) \leqslant \omega(\delta) \leqslant \omega(\delta+\sigma) \leqslant \omega(\delta)+\omega(\sigma) \text { при } \delta, \sigma \in[0, \infty) .
$$


Тогда для области $D \subset \mathbb{R}^{d}, l \in \mathbb{Z}_{+}, 1 \leqslant p \leqslant \infty$, и $\omega$ через $F_{p}^{l, \omega}(D)$ обозначим множество функций $f \in W_{p}^{l}(D)$, для которых выполняется неравенство

$$
\sum_{\lambda \in \mathbb{Z}_{+l}^{\prime d}} \sup _{h \in \mathbb{R}^{d} \backslash\{0\}} \operatorname{vrai} \frac{1}{\omega(\|h\|)}\left\|\mathscr{D}^{\lambda} f(x+h)-\mathscr{D}^{\lambda} f(x)\right\|_{L_{p}\left(D_{h}\right)} \leqslant 1,
$$

где $D_{h}=\{x \in D: x+t h \in D \forall t \in[0,1]\}$.

Для $l \in \mathbb{Z}_{+}, d \in \mathbb{N}$ и измеримого по Лебегу множества $Q$, лежащего в ограниченной области $D \subset \mathbb{R}^{d}$, мера которого mes $Q>0$, обозначим через

$$
T_{Q}^{l, d}: W_{1}^{l}(D) \rightarrow \mathscr{P}_{l, d}
$$

линейный оператор, определяемый для $f \in W_{1}^{l}(D)$ равенством

$$
\left(T_{Q}^{l, d} f\right)(x)=\frac{1}{\operatorname{mes} Q} \sum_{\lambda \in \mathbb{Z}_{+l}^{d}} \frac{1}{\lambda !} \int_{Q} \mathscr{D}^{\lambda} f(y)(x-y)^{\lambda} d y, \quad \lambda !=\lambda_{1} ! \cdots \lambda_{d} ! .
$$

Положим также

$$
T_{Q}^{-1, d} f=0, \quad f \in L_{1}(Q)
$$

В рассматриваемой ситуации наряду с оператором $T_{Q}^{l, d}$ определим линейный оператор $T_{Q}^{l l, d}: W_{1}^{l}(D) \rightarrow \mathscr{P}_{l, d}$, полагая

$$
T_{Q}^{l l, d} f=T_{Q}^{l, d} f+T_{Q}^{l-1, d} f-T_{Q}^{l-1, d} T_{Q}^{l, d} f, \quad f \in W_{1}^{l}(D) .
$$

Лемма 1.1.1. Пусть $l \in \mathbb{Z}_{+}, d \in \mathbb{N}, 1 \leqslant p \leqslant \infty, D$ - ограниченная область в $\mathbb{R}^{d}$ и $Q \subset D$ - измеримое по Лебегу множество, для которого mes $Q>0$. Тогда для $f \in W_{p}^{l}(D)$ и $\lambda \in \mathbb{Z}_{+l}^{d}$ справедливо равенство

$$
\mathscr{D}^{\lambda} T_{Q}^{\prime l, d} f=T_{Q}^{\prime l-|\lambda|, d} \mathscr{D}^{\lambda} f .
$$

Для формулирования теоремы 1.1.2 понадобятся следующие обозначения.

Через $I^{d} \subset \mathbb{R}^{d}$ будем обозначать куб

$$
I^{d}=\left\{x \in \mathbb{R}^{d}: 0<x_{j}<1, j=1, \ldots, d\right\} .
$$

Для $r, d \in \mathbb{N}$ через $\mathrm{N}_{r}^{d}$ обозначим множество мультииндексов

$$
\mathrm{N}_{r}^{d}=\left\{\nu \in \mathbb{Z}^{d}: 0 \leqslant \nu_{j} \leqslant r-1, j=1, \ldots, d\right\} .
$$

Для $k \in \mathbb{Z}_{+}, d \in \mathbb{N}, \nu \in \mathrm{N}_{2^{k}}^{d}$ определим кубы

$$
\begin{aligned}
& Q_{k, \nu}^{d}=\left\{x \in \mathbb{R}^{d}: 2^{-k} \nu_{j}<x_{j}<2^{-k}\left(\nu_{j}+1\right), j=1, \ldots, d\right\}, \\
& Q_{k, \nu}^{\prime d}=\left\{x \in \mathbb{R}^{d}: 2^{-k}\left(\nu_{j}+\frac{1}{4}\right) \leqslant x_{j} \leqslant 2^{-k}\left(\nu_{j}+\frac{3}{4}\right), j=1, \ldots, d\right\},
\end{aligned}
$$

а через $\chi_{k, \nu}^{d}$ обозначим характеристическую функцию множества $Q_{k, \nu}^{d}$.

Для $k, l \in \mathbb{Z}_{+}, d \in \mathbb{N}$ определим линейньй оператор $U_{k}^{l, d}: W_{1}^{l}\left(I^{d}\right) \rightarrow L_{\infty}\left(I^{d}\right)$, полагая

$$
U_{k}^{l, d} f=\sum_{\nu \in \mathrm{N}_{2^{k}}^{d}}\left(T_{Q_{k, \nu}^{\prime l d}}^{l, d} f\right) \cdot \chi_{k, \nu}^{d}, \quad f \in W_{1}^{l}\left(I^{d}\right)
$$


ТЕОРема 1.1.2. Пусть $l \in \mathbb{Z}_{+}, d \in \mathbb{N}, 1 \leqslant p, q \leqslant \infty u \omega-$ модуль непрерывности таковы, что при $p<q$ существует константа $c_{1}(l, d, p, q, \omega)>0$ такая, что для $\delta \in[0,1]$ соблюдается неравенство

$$
\int_{0}^{1} t^{l-d / p+d / q-1} \omega(\delta t) d t \leqslant c_{1} \omega(\delta) .
$$

Тогда существует константа $c_{2}(l, d, p, q, \omega)>0$ такая, что для $f \in F_{p}^{l, \omega}\left(I^{d}\right)$ при $k \in \mathbb{Z}_{+}$выполняется неравенство

$$
\left\|f-U_{k}^{l, d} f\right\|_{L_{q}\left(I^{d}\right)} \leqslant c_{2} 2^{-k\left(l-(d / p-d / q)_{+}\right)} \omega\left(2^{-k}\right)
$$

әде, как обично, $\tau_{+}=\frac{1}{2}(\tau+|\tau|)$.

Существенную роль в доказательстве основного результата $\S 1$ играет

Теорема 1.1.3. Пусть $l \in \mathbb{Z}_{+}, d \in \mathbb{N}, 1 \leqslant s, q \leqslant \infty, \lambda \in \mathbb{Z}_{+l}^{d}, \rho>0$. Тогда существует семейство констант

$$
\left\{c_{3}(l, d, \lambda, \rho)>0, l \in \mathbb{Z}_{+}, d \in \mathbb{N}, \lambda \in \mathbb{Z}_{+l}^{d}, \rho>0\right\}
$$

такое, что для любого ограниченного измеримого по Лебегу множества $Q \subset \mathbb{R}^{d}$ и любого $\delta>0$, для которых существует точка $x^{0} \in \mathbb{R}^{d}$ такая, что $x^{0}+\delta I^{d} \subset Q$, существует линейный оператор

$$
P_{Q, \delta, x^{0}}^{l, d}: L_{1}(Q) \rightarrow \mathscr{P}_{l, d}
$$

обладающий следующими свойствами:

1) для любого измеримого по Лебегу мноэсества $D \subset \mathbb{R}^{d}$ такого, что

$$
\sup _{x \in D}\left\|x-x^{0}\right\| \leqslant \rho \delta
$$

для $f \in L_{s}(Q)$ при $\lambda \in \mathbb{Z}_{+l}^{d}$ справедливо неравенство

$$
\left\|\mathscr{D}^{\lambda} P_{Q, \delta, x^{0}}^{l, d} f\right\|_{L_{q}(Q)} \leqslant c_{3} \delta^{-|\lambda|-d / s+d / q}\|f\|_{L_{s}(Q)}
$$

2) для $f \in \mathscr{P}_{l, d}$ имеет место равенство

$$
P_{Q, \delta, x^{0}}^{l, d}\left(\left.f\right|_{Q}\right)=f
$$

1.2. В этом пункте мы получим оценку, о которой сказано в названии $\S 1$. Но прежде напомним постановку интересующей нас задачи.

Как отмечалось выше, задача о приближении линейного оператора частного дифференцирования ограниченными операторами является частным случаем задачи о приближении неограниченного линейного оператора ограниченными, которая формулируется следующим образом.

Пусть $X, Y$ - банаховы пространства, $B(X, Y)$ - банахово пространство ограниченных линейных операторов $V: X \rightarrow Y$ с обьчной нормой

$$
\|V\|_{B(X, Y)}=\sup _{\left\{x \in X:\|x\|_{X} \leqslant 1\right\}}\|V x\|_{Y}
$$

$A: D(A) \rightarrow Y-$ линейный оператор с областью определения $D(A) \subset X$. Пусть еше множество $K \subset D(A)$ и $\rho>0$. Требуется описать поведение в зависимости от $\rho$ величины

$$
\mathbf{E}(A, X, Y, K, \rho)=\inf _{\left\{V \in B(X, Y):\|V\|_{B(X, Y)} \leqslant \rho\right\}} \sup _{x \in K}\|A x-V x\|_{Y} .
$$


Tеорема 1.2.1. Пусть $l \in \mathbb{Z}_{+}, d \in \mathbb{N}, 1 \leqslant p, q, s \leqslant \infty, \omega-$ модуль непрервьвности, $\lambda \in \mathbb{Z}_{+l}^{d}, \theta=|\lambda|+(d / s-d / q)_{+}, \gamma=l-|\lambda|-(d / p-d / q)_{+}$. Пусть eще $X=L_{s}\left(I^{d}\right), Y=L_{q}\left(I^{d}\right), A=\mathscr{D}^{\lambda}, D(A)=\left\{f \in L_{s}\left(I^{d}\right): \mathscr{D}^{\lambda} f \in L_{q}\left(I^{d}\right)\right\}$, $K=F_{p}^{l, \omega}\left(I^{d}\right), \theta>0$ и при $s>p$ имеет место включение

$$
t^{l-d / p+d / s-1} \omega(t) \in L_{1}(I)
$$

а при $q>p$ для $\delta \in[0,1]$ выполняется неравенство

$$
\int_{0}^{1} t^{\gamma-1} \omega(\delta t) d t \leqslant c_{1}(l-|\lambda|, d, p, q, \omega) \omega(\delta) .
$$

Тогда существуют константы $\rho_{1}(l, d, q, s, \lambda)>0$ u $c_{4}(l, d, p, q, s, \omega, \lambda)>0$ такие, что для $\rho>\rho_{1}$ справедливо неравенство

$$
\mathbf{E}(A, X, Y, K, \rho) \leqslant c_{4} \rho^{-\gamma / \theta} \omega\left(\rho^{-1 / \theta}\right) .
$$

Доказательство теоремы 1.2.1, по сушеству, сводится к доказательству леммы 1.2.2.

ЛЕмма 1.2.2. Пусть выполнень условия теоремы 1.2.1. Тогда существуют константы $c_{5}(l, d, p, q, \omega, \lambda)>0$ и $c_{6}(l, d, q, s, \lambda)>0$ такие, что при $k \in \mathbb{Z}_{+}$ для операторов $V_{\lambda, k}^{l, d}: L_{1}\left(I^{d}\right) \rightarrow L_{\infty}\left(I^{d}\right)$, определяемых равенствами

$$
V_{\lambda, k}^{l, d}=\sum_{\nu \in \mathrm{N}_{2}^{d}}\left(\mathscr{D}^{\lambda} S_{k, \nu}^{l, d} f\right) \chi_{k, \nu}^{d}
$$

где $S_{k, \nu}^{l, d}=P_{Q, \delta, x^{0}}^{l, d}$ при $Q=Q_{k, \nu}^{d}, \delta=2^{-k}, x^{0}=2^{-k} \nu$, соблюдаются неравенства

$$
\begin{aligned}
\left\|\mathscr{D}^{\lambda} f-V_{\lambda, k}^{l, d} f\right\|_{L_{q}\left(I^{d}\right)} \leqslant c_{5} 2^{-k \gamma} \omega\left(2^{-k}\right), & f \in F_{p}^{l, \omega}\left(I^{d}\right) \\
\left\|V_{\lambda, k}^{l, d} f\right\|_{L_{q}\left(I^{d}\right)} \leqslant c_{6} 2^{k \theta}\|f\|_{L_{s}\left(I^{d}\right)}, & f \in L_{s}\left(I^{d}\right) .
\end{aligned}
$$

ДокаЗАТЕльство. Доказательство леммы мы проведем при $q \neq \infty$, однако при несушественной корректировке в выкладках оно сохраняет свою силу и при $q=\infty$.

Прежде всего отметим, что в силу неравенства Гёльдера при $s \leqslant p$ и в силу (1.2.1) при $s>p$ (см. [6]) имеет место включение $F_{p}^{l, \omega}\left(I^{d}\right) \subset L_{s}\left(I^{d}\right)$.

Далее, поскольку для $f \in F_{p}^{l, \omega}\left(I^{d}\right)$ и $\lambda \in \mathbb{Z}_{+l}^{d}$ производная $\mathscr{D}^{\lambda} f \in F_{p}^{l-|\lambda|, \omega}\left(I^{d}\right)$, то, принимая во внимание (1.2.2), а также предыдушее замечание, заключаем, что $K \subset D(A)$ (см. теорему 1.1.2) и при $k \in \mathbb{Z}_{+}$для $f \in K$ выполняется неравенство

$$
\left\|\mathscr{D}^{\lambda} f-V_{\lambda, k}^{l, d} f\right\|_{Y} \leqslant\left\|\mathscr{D}^{\lambda} f-U_{k}^{l-|\lambda|, d} \mathscr{D}^{\lambda} f\right\|_{Y}+\left\|U_{k}^{l-|\lambda|, d} \mathscr{D}^{\lambda} f-V_{\lambda, k}^{l, d} f\right\|_{Y} .
$$

Из (1.1.3) с учетом (1.1.2) и (1.2.2) для $f \in K$ при $k \in \mathbb{Z}_{+}$следует неравенство

$$
\left\|\mathscr{D}^{\lambda} f-U_{k}^{l-|\lambda|, d} \mathscr{D}^{\lambda} f\right\|_{Y} \leqslant c_{2} 2^{-k \gamma} \omega\left(2^{-k}\right) .
$$


Для оценки второго слагаемого в правой части (1.2.6), используя (1.1.1), (1.1.5) и (1.1.4), получаем

$$
\begin{aligned}
& \left\|U_{k}^{l-|\lambda|, d} \mathscr{D}^{\lambda} f-V_{\lambda, k}^{l, d} f\right\|_{L_{q}\left(I^{d}\right)}^{q}=\sum_{\nu \in \mathrm{N}_{2^{d}}}\left\|T_{Q_{k, \nu}^{\prime l}}^{l-|\lambda|, d} \mathscr{D}^{\lambda} f-\mathscr{D}^{\lambda} S_{k, \nu}^{l, d} f\right\|_{L_{q}\left(Q_{k, \nu}^{d}\right)}^{q} \\
& =\sum_{\nu \in \mathrm{N}_{2^{k}}}\left\|\mathscr{D}^{\lambda} T_{Q_{k, \nu}^{\prime d}}^{\prime l, d} f-\mathscr{D}^{\lambda} S_{k, \nu}^{l, d} f\right\|_{L_{q}\left(Q_{k, \nu}^{d}\right)}^{q} \\
& =\sum_{\nu \in \mathrm{N}_{2^{k}}^{d}}\left\|\mathscr{D}^{\lambda} S_{k, \nu}^{l, d}\left(T_{Q_{k, \nu}^{\prime d}}^{l, d} f-f\right)\right\|_{L_{q}\left(Q_{k, \nu}^{d}\right)}^{q} \\
& \leqslant \sum_{\nu \in \mathrm{N}_{2^{k}}^{k}}\left(c_{3} 2^{k|\lambda|}\left\|T_{Q_{k, \nu}^{\prime l}}^{\prime l, d} f-f\right\|_{L_{q}\left(Q_{k, \nu}^{d}\right.}\right)^{q} \\
& =\left(c_{3} \cdot 2^{k|\lambda|}\right)^{q}\left\|f-U_{k}^{l, d} f\right\|_{L_{q}\left(I^{d}\right)}^{q} .
\end{aligned}
$$

Откуда, снова обрашаясь к (1.1.3), выводим

$$
\left\|U_{k}^{l-|\lambda|, d} \mathscr{D}^{\lambda} f-V_{\lambda, k}^{l, d} f\right\|_{L_{q}\left(I^{d}\right)} \leqslant c_{7} \cdot 2^{-k \gamma} \cdot \omega\left(2^{-k}\right), \quad f \in K, \quad k \in \mathbb{Z}_{+} .
$$

Объединяя (1.2.6), (1.2.7) и (1.2.8), приходим к (1.2.4).

Теперь, если $q \geqslant s$, то, применяя (1.1.4) и неравенство Гёльдера с показателем $s / q \leqslant 1$, имеем для $f \in L_{s}\left(I^{d}\right)$ :

$$
\begin{aligned}
\left\|V_{\lambda, k}^{l, d} f\right\|_{L_{q}\left(I^{d}\right)}^{q} & =\sum_{\nu \in \mathrm{N}_{2^{k}}}\left\|\mathscr{D}^{\lambda} S_{k, \nu}^{l, d} f\right\|_{L_{q}\left(Q_{k, \nu}^{d}\right)}^{q} \\
& \leqslant \sum_{\nu \in \mathrm{N}_{2^{k}}}\left(c_{3} 2^{k(|\lambda|+d / s-d / q)}\|f\|_{L_{s}\left(Q_{k, \nu}^{d}\right)}\right)^{q} \\
& \leqslant\left(c_{3} 2^{k(|\lambda|+d / s-d / q)}\right)^{q}\left(\sum_{\nu \in \mathrm{N}^{d}}\|f\|_{2_{s}\left(Q_{k, \nu}^{d}\right)}^{s}\right)^{q / s} \\
& =\left(c_{3} 2^{k(|\lambda|+d / s-d / q)}\right)^{q}\|f\|_{L_{s}\left(I^{d}\right)}^{q} .
\end{aligned}
$$

Откуда вытекает (1.2.5) при $q \geqslant s$.

Если же $q<s$, то, пользуясь неравенством Гёльдера и соотношением (1.2.5) при $q=s$, находим, что

$$
\left\|V_{\lambda, k}^{l, d} f\right\|_{L_{q}\left(I^{d}\right)} \leqslant\left\|V_{\lambda, k}^{l, d} f\right\|_{L_{s}\left(I^{d}\right)} \leqslant c_{6} 2^{k|\lambda|}\|f\|_{L_{s}\left(I^{d}\right)}, \quad f \in L_{s}\left(I^{d}\right),
$$

т.е. (1.2.5) справедливо и при $q<s$.

Чтобы получить (1.2.3), достаточно для $\rho>\rho_{1}=c_{6}$ выбрать $k \in \mathbb{Z}_{+}$так, чтобы вьполнялось неравенство

$$
c_{6} 2^{k \theta} \leqslant \rho<c_{6} 2^{(k+1) \theta},
$$

и проверить, пользуясь $(1.2 .9),(1.2 .5)$ и (1.2.4), что $\left\|V_{\lambda, k}^{l, d}\right\|_{B(X, Y)} \leqslant \rho$ и

$$
\sup _{f \in K}\left\|\mathscr{D}^{\lambda} f-V_{\lambda, k}^{l, d} f\right\|_{Y} \leqslant c_{4} \rho^{-\gamma / \theta} \omega\left(\rho^{-1 / \theta}\right) .
$$


$\S 2$. Оценка снизу величины $\mathbf{E}\left(\mathscr{D}^{\lambda}, L_{s}\left(I^{d}\right), L_{q}\left(I^{d}\right), F_{p}^{l, \omega}\left(I^{d}\right), \rho\right)$

2.1. В этом пункте будет доказано наиболее важное вспомогательное утверждение, необходимое для получения основного результата $\S 2$.

Всюду ниже наряду с обозначениями, применявшимися в $\S 1$, будем использовать также следующие.

Для $n \in \mathbb{N}, 1 \leqslant p \leqslant \infty$, как обычно, через $\ell_{p}^{n}$ обозначается пространство $\mathbb{R}^{n}$ с фиксированной в нем нормой

$$
\|x\|_{\ell_{p}^{n}}= \begin{cases}\left(\sum_{j=1}^{n}\left|x_{j}\right|^{p}\right)^{1 / p}, & p<\infty, \\ \max _{j=1, \ldots, n}\left|x_{j}\right|, & p=\infty, x \in \mathbb{R}^{n} .\end{cases}
$$

Через $\varepsilon_{i}, i=1, \ldots, n$, обозначим вектор $\varepsilon_{i}=\left(0, \ldots, 0,1_{i}, 0, \ldots, 0\right)$.

Через $E$ будем обозначать тождественный оператор в $\mathbb{R}^{n}$.

Для доказательства основного результата этого пункта, дающего значение величины $\mathbf{E}(A, X, Y, K, \rho)$ при

$$
A=E, \quad X=\ell_{s}^{n}, \quad Y=\ell_{q}^{n}, \quad K=\left\{x \in \mathbb{R}^{n}:\|x\|_{\ell_{p}^{n}} \leqslant 1\right\}
$$

понадобятся следующие леммы.

ЛЕмма 2.1.1. Пусть $\left\{a_{i j} \in \mathbb{R}, i, j,=1, \ldots, n\right\}$ - матрица линейного операmора $U: \mathbb{R}^{n} \rightarrow \mathbb{R}^{n}$ в базисе $\left\{\varepsilon_{i}, i=1, \ldots, n\right\}$. Тогда

$$
\|U\|_{B\left(\ell_{1}^{n}, \ell_{\infty}^{n}\right)}=\max _{i, j=1, \ldots, n}\left|a_{i j}\right| .
$$

ДОКАЗАТЕЛЬСТвО. Для $x=\left(x_{1}, \ldots, x_{n}\right)=\sum_{j=1}^{n} x_{j} \varepsilon_{j}$ имеем

$$
\begin{aligned}
\|U x\|_{\ell_{\infty}^{n}} & =\left\|\sum_{i=1}^{n}\left(\sum_{j=1}^{n} a_{i j} x_{j}\right) \varepsilon_{i}\right\|_{\ell_{\infty}^{n}}=\max _{i=1, \ldots, n}\left|\sum_{j=1}^{n} a_{i j} x_{j}\right| \\
& \leqslant \max _{i=1, \ldots, n}\left(\|x\|_{\ell_{1}^{n}} \max _{j=1, \ldots, n}\left|a_{i j}\right|\right)=\|x\|_{\ell_{1}^{n}} \max _{i=1, \ldots, n}\left(\max _{j=1, \ldots, n}\left|a_{i j}\right|\right) \\
& =\left(\max _{i, j=1, \ldots, n}\left|a_{i j}\right|\right)\|x\|_{\ell_{1}^{n} .}
\end{aligned}
$$

$\mathrm{C}$ другой стороны, если для $1 \leqslant i_{0}, j_{0} \leqslant n$ имеет место равенство $\left|a_{i_{0}, j_{0}}\right|=$ $\max _{i, j=1, \ldots, n}\left|a_{i j}\right|$, то, учитьвая, что $\left\|\varepsilon_{j_{0}}\right\|_{\ell_{1}^{n}}=1$, получаем:

$$
\|U\|_{B\left(\ell_{1}^{n}, \ell_{\infty}^{n}\right)} \geqslant\left\|U \varepsilon_{j_{0}}\right\|_{\ell_{\infty}^{n}}=\max _{i=1, \ldots, n}\left|a_{i, j_{0}}\right| \geqslant\left|a_{i_{0}, j_{0}}\right| .
$$

Объединяя сказанное, убеждаемся в справедливости (2.1.1).

ЛЕмма 2.1.2. При любом $n \in \mathbb{N}$ для любой вещественной матриць $\left\{b_{i j}, i, j=1, \ldots, n\right\}$ такой, что для каждого $i=1, \ldots, n$ әлемент $b_{i i} \leqslant 0$, существует вектор $\xi=\left(\xi_{1}, \ldots, \xi_{n}\right) \in \mathbb{R}^{n}$ такой, что

$$
\begin{gathered}
\left|\xi_{i}\right|=1, \quad i=1, \ldots, n, \\
\sum_{i, j=1}^{n} b_{i j} \xi_{i} \xi_{j} \leqslant 0 .
\end{gathered}
$$


ДОКАЗАТЕЛЬСТВО проведем по индукции относительно $n$. При $n=1$ утверждение леммы тривиально. Предположим, что утверждение леммы справедливо при $n=k, k \in \mathbb{N}$. Покажем, что тогда оно имеет место и при $n=k+1$.

Пусть $\left\{b_{i j}, i, j=1, \ldots, k+1\right\}$ - вещественная матрица, для которой $b_{i i} \leqslant 0$, $i=1, \ldots, k+1$. Исходя из предположения индукции, выберем для матрицы $\left\{b_{i j}, i, j=1, \ldots, k\right\}$ вектор $\xi^{\prime}=\left(\xi_{1}, \ldots, \xi_{k}\right) \in \mathbb{R}^{k}$ так, чтобы выполнялись соотношения (2.1.2) и (2.1.3) при $n=k$. Положим

$$
\xi_{k+1}=\left\{\begin{array}{l}
1, \quad \text { если } \sum_{i=1}^{k}\left(b_{i, k+1}+b_{k+1, i}\right) \xi_{i} \leqslant 0 \\
-1, \quad \text { если } \sum_{i=1}^{k}\left(b_{i, k+1}+b_{k+1, i}\right) \xi_{i}>0 .
\end{array}\right.
$$

Тогда для $\xi=\left(\xi_{1}, \ldots, \xi_{k}, \xi_{k+1}\right) \in \mathbb{R}^{k+1}$ соблюдается (2.1.2) при $n=k+1$ и

$$
\begin{aligned}
\sum_{i, j=1}^{k+1} b_{i j} \xi_{i} \xi_{j} & =\sum_{i, j=1}^{k} b_{i j} \xi_{i} \xi_{j}+\sum_{i=1}^{k} b_{i, k+1} \xi_{i} \xi_{k+1}+\sum_{i=1}^{k} b_{k+1, i} \xi_{k+1} \xi_{i}+b_{k+1, k+1} \xi_{k+1}^{2} \\
& =\sum_{i, j=1}^{k} b_{i j} \xi_{i} \xi_{j}+\xi_{k+1}\left(\sum_{i=1}^{k}\left(b_{i, k+1}+b_{k+1, i}\right) \xi_{i}\right)+b_{k+1, k+1} \xi_{k+1}^{2} \leqslant 0
\end{aligned}
$$

т.е. имеет место (2.1.3) при $n=k+1$.

ЛЕмма 2.1.3. При любом $n \in \mathbb{N}$ для любого линейного оператора $T: \mathbb{R}^{n} \rightarrow \mathbb{R}^{n}$, матрица которого $\left\{b_{i j}, i, j=1, \ldots, n\right\}$ в базисе $\left\{\varepsilon_{i}, i=1, \ldots, n\right\}$ обладает тем свойством, что для каждого $i=1, \ldots, n$ әлемент $b_{i i} \leqslant 0$, справедливо неравенство

$$
\|E-T\|_{B\left(\ell_{\infty}^{n}, \ell_{1}^{n}\right)} \geqslant n
$$

ДокАЗАТЕЛЬСТво. В соответствии с леммой 2.1.2 возьмем вектор $\xi=$ $\left(\xi_{1}, \ldots, \xi_{n}\right) \in \mathbb{R}^{n}$ такой, что для матрицы $\left\{b_{i j}, i, j=1, \ldots, n\right\}$ (матрицы оператора $T$ в базисе $\left.\left\{\varepsilon_{i}, i=1, \ldots, n\right\}\right)$ и вектора $\xi$ соблюдаются условия (2.1.2) и (2.1.3). Тогда имеем:

$$
\begin{aligned}
n & =\sum_{i=1}^{n} \xi_{i}^{2} \leqslant \sum_{i=1}^{n} \xi_{i}^{2}-\sum_{i, j=1}^{n} b_{i j} \xi_{i} \xi_{j}=\sum_{i=1}^{n}\left(\xi_{i}^{2}-\sum_{j=1}^{n} b_{i j} \xi_{i} \xi_{j}\right) \\
& =\sum_{i=1}^{n} \xi_{i}\left(\xi_{i}-\sum_{j=1}^{n} b_{i j} \xi_{j}\right) \leqslant \sum_{i=1}^{n}\left|\xi_{i}\left(\xi_{i}-\sum_{j=1}^{n} b_{i j} \xi_{j}\right)\right|=\sum_{i=1}^{n}\left|\xi_{i}\right| \cdot\left|\xi_{i}-\sum_{j=1}^{n} b_{i j} \xi_{j}\right| \\
& =\sum_{i=1}^{n}\left|\xi_{i}-\sum_{j=1}^{n} b_{i j} \xi_{j}\right|=\|\xi-T \xi\|_{\ell_{1}^{n}}=\|(E-T) \xi\|_{\ell_{1}^{n}} \\
& \leqslant\|E-T\|_{B\left(\ell_{\infty}^{n}, \ell_{1}^{n}\right)}\|\xi\|_{\ell_{\infty}^{n}}=\|E-T\|_{B\left(\ell_{\infty}^{n}, \ell_{1}^{n}\right)} .
\end{aligned}
$$

Обозначая через $\mathscr{L}_{n}, n \in \mathbb{N}$, множество всех линейных операторов $U: \mathbb{R}^{n} \rightarrow \mathbb{R}^{n}$, с помошью лемм 2.1.1 и 2.1.3 установим справедливость теоремы 2.1.4. 
ТЕОРема 2.1.4. При любом $n \in \mathbb{N}$ и любом $\varepsilon \in I$ имеет место равенство

$$
\min \left\{\|E-U\|_{B\left(\ell_{\infty}^{n}, \ell_{1}^{n}\right)}: U \in \mathscr{L}_{n},\|U\|_{B\left(\ell_{1}^{n}, \ell_{\infty}^{n}\right)} \leqslant \varepsilon\right\}=(1-\varepsilon) n .
$$

ДокаЗАтельство. Пусть $U \in \mathscr{L}_{n}$ и $\|U\|_{B\left(\ell_{1}^{n}, \ell_{\infty}^{n}\right)} \leqslant \varepsilon$. Тогда, учитывая $(2.1 .1)$, нетрудно видеть, что для оператора $T=(1-\varepsilon)^{-1}(U-\varepsilon E)$ соблюдены условия леммы 2.1.3. Поэтому, пользуясь оценкой (2.1.4), получаем

$$
\begin{aligned}
\|E-U\|_{B\left(\ell_{\infty}^{n}, \ell_{1}^{n}\right)} & =\|E-\varepsilon E+\varepsilon E-U\|_{B\left(\ell_{\infty}^{n}, \ell_{1}^{n}\right)} \\
& =\|(1-\varepsilon) E-(U-\varepsilon E)\|_{B\left(\ell_{\infty}^{n}, \ell_{1}^{n}\right)} \\
& =(1-\varepsilon)\left\|E-(1-\varepsilon)^{-1}(U-\varepsilon E)\right\|_{B\left(\ell_{\infty}^{n}, \ell_{1}^{n}\right)} \geqslant(1-\varepsilon) n .
\end{aligned}
$$

Кроме того, для оператора $\varepsilon E \in \mathscr{L}_{n}$ его норма

$$
\|\varepsilon E\|_{B\left(\ell_{1}^{n}, \ell_{\infty}^{n}\right)}=\varepsilon
$$

a

$$
\|E-\varepsilon E\|_{B\left(\ell_{\infty}^{n}, \ell_{1}^{n}\right)}=(1-\varepsilon) n .
$$

Сопоставляя сказанное, заключаем, что имеет место (2.1.5).

Из теоремы 2.1.4 вытекает

ТЕОРема 2.1.5. При любом $n \in \mathbb{N}$ и любом $\varepsilon \in I$ справедливо равенство

$$
\min \left\{\|E-U\|_{B\left(\ell_{1}^{n}, \ell_{\infty}^{n}\right)}: U \in \mathscr{L}_{n},\|U\|_{B\left(\ell_{\infty}^{n}, \ell_{1}^{n}\right)} \leqslant(1-\varepsilon) n\right\}=\varepsilon .
$$

ДоказАТЕЛьство. Подставляя в (2.1.5) $E-U$ вместо $U$, находим

$$
\min \left\{\|U\|_{B\left(\ell_{\infty}^{n}, \ell_{1}^{n}\right)}: U \in \mathscr{L}_{n},\|E-U\|_{B\left(\ell_{1}^{n}, \ell_{\infty}^{n}\right)} \leqslant \varepsilon\right\}=(1-\varepsilon) n .
$$

Равенство (2.1.7) позволяет утверждать, что для любого оператора $U \in \mathscr{L}_{n}$ такого, что

вьполняется неравенство

$$
\|U\|_{B\left(\ell_{\infty}^{n}, \ell_{1}^{n}\right)}<(1-\varepsilon) n
$$

$$
\|E-U\|_{B\left(\ell_{1}^{n}, \ell_{\infty}^{n}\right)}>\varepsilon
$$

ибо если для некоторого $U \in \mathscr{L}_{n},\|U\|_{B\left(\ell_{\infty}^{n}, \ell_{1}^{n}\right)}<(1-\varepsilon) n$, имеет место

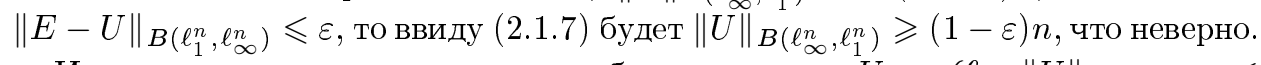

Из сказанного следует, что для любого оператора $U \in \mathscr{L}_{n},\|U\|_{B\left(\ell_{\infty}^{n}, \ell_{1}^{n}\right)} \leqslant$ $(1-\varepsilon) n$, при любом $k \in \mathbb{N}$ соблюдается неравенство

$$
\left\|E-\left(1-k^{-1}\right) U\right\|_{B\left(\ell_{1}^{n}, \ell_{\infty}^{n}\right)}>\varepsilon .
$$

Переходя в этом неравенстве к пределу при $k \rightarrow \infty$, получаем, что для любого $U \in \mathscr{L}_{n},\|U\|_{B\left(\ell_{\infty}^{n}, \ell_{1}^{n}\right)} \leqslant(1-\varepsilon) n$, вьполняется неравенство

$$
\|E-U\|_{B\left(\ell_{1}^{n}, \ell_{\infty}^{n}\right)} \geqslant \varepsilon .
$$

Отсюда, учитывая, что для оператора $(1-\varepsilon) E \in \mathscr{L}_{n}$ имеют место равенства

$$
\|(1-\varepsilon) E\|_{B\left(\ell_{\infty}^{n}, \ell_{1}^{n}\right)}=(1-\varepsilon) n, \quad\|E-(1-\varepsilon) E\|_{B\left(\ell_{1}^{n}, \ell_{\infty}^{n}\right)}=\varepsilon
$$

следует справедливость (2.1.6).

С использованием теорем 2.1.4 и 2.1.5 может быть доказана 
Теорема 2.1.6. При любых $n \in \mathbb{N}, \varepsilon \in I, 1 \leqslant p, q, s \leqslant \infty$ выполняется равенство

$\min \left\{\|E-U\|_{B\left(\ell_{p}^{n}, \ell_{q}^{n}\right)}: U \in \mathscr{L}_{n},\|U\|_{B\left(\ell_{s}^{n}, \ell_{q}^{n}\right)} \leqslant \varepsilon n^{(1 / q-1 / s)_{+}}\right\}=(1-\varepsilon) n^{(1 / q-1 / p)_{+}}$.

ДокАЗАТЕльство. Сначала покажем, что для каждого оператора $U \in \mathscr{L}_{n}$, для которого

$$
\|U\|_{B\left(\ell_{s}^{n}, \ell_{q}^{n}\right)} \leqslant \varepsilon n^{(1 / q-1 / s)_{+}},
$$

соблюдается неравенство

$$
\|E-U\|_{B\left(\ell_{p}^{n}, \ell_{q}^{n}\right)} \geqslant(1-\varepsilon) n^{(1 / q-1 / p)_{+}} .
$$

При этом рассмотрим четыре случая соотношений между $p, q$ и $s$.

1) $q \leqslant p, s$.

В этом случае, учитьвая, что для вектора $\xi=n^{-1 / p} \sum_{j=1}^{n} \varepsilon_{j}$ его норма $\|\xi\|_{\ell_{p}^{n}}=$ 1 , для любого оператора $U \in \mathscr{L}_{n}$, удовлетворяюшего условию (2.1.9), имеем:

$$
\begin{aligned}
\| E & -U\left\|_{B\left(\ell_{p}^{n}, \ell_{q}^{n}\right)} \geqslant\right\| \xi-U \xi\left\|_{\ell_{q}^{n}} \geqslant\right\| \xi\left\|_{\ell_{q}^{n}}-\right\| U \xi \|_{\ell_{q}^{n}} \\
& \geqslant\|\xi\|_{\ell_{q}^{n}}-\|U\|_{B\left(\ell_{s}^{n}, \ell_{q}^{n}\right)}\|\xi\|_{\ell_{s}^{n}} \geqslant\|\xi\|_{\ell_{q}^{n}}-\varepsilon n^{1 / q-1 / s}\|\xi\|_{\ell_{s}^{n}} \\
& =n^{1 / q-1 / p}-\varepsilon n^{1 / q-1 / s} n^{1 / s-1 / p}=(1-\varepsilon) n^{(1 / q-1 / p)_{+} .}
\end{aligned}
$$

2) $q \geqslant p, s$.

В этом случае, беря любой из векторов $\varepsilon_{j}, j=1, \ldots, n$, для каждого оператора $U \in \mathscr{L}_{n}$, удовлетворяющего (2.1.9), получаем:

$$
\begin{aligned}
\|E-U\|_{B\left(\ell_{p}^{n}, \ell_{q}^{n}\right)} & \geqslant\left\|\varepsilon_{j}-U \varepsilon_{j}\right\|_{\ell_{q}^{n}} \geqslant\left\|\varepsilon_{j}\right\|_{\ell_{q}^{n}}-\left\|U \varepsilon_{j}\right\|_{\ell_{q}^{n}} \\
& \geqslant\left\|\varepsilon_{j}\right\|_{\ell_{q}^{n}}-\|U\|_{B\left(\ell_{s}^{n}, \ell_{q}^{n}\right)}\left\|\varepsilon_{j}\right\|_{\ell_{s}^{n}} \geqslant\left\|\varepsilon_{j}\right\|_{\ell_{q}^{n}}-\varepsilon\left\|\varepsilon_{j}\right\|_{\ell_{s}^{n}}=1-\varepsilon
\end{aligned}
$$

что совпадает с (2.1.10).

3) $s<q<p$.

В этом случае, пользуясь тем, что для любого оператора $U \in \mathscr{L}_{n}$, удовлетворяющего условию (2.1.9), соблюдается неравенство

$$
\|U\|_{B\left(\ell_{1}^{n}, \ell_{\infty}^{n}\right)} \leqslant\|E\|_{B\left(\ell_{q}^{n}, \ell_{\infty}^{n}\right)}\|U\|_{B\left(\ell_{s}^{n}, \ell_{q}^{n}\right)}\|E\|_{B\left(\ell_{1}^{n}, \ell_{s}^{n}\right)} \leqslant\|U\|_{B\left(\ell_{s}^{n}, \ell_{q}^{n}\right)} \leqslant \varepsilon
$$

заключаем, что

$$
\begin{array}{r}
\min \left\{\|E-U\|_{B\left(\ell_{p}^{n}, \ell_{q}^{n}\right)}: U \in \mathscr{L}_{n},\|U\|_{B\left(\ell_{s}^{n}, \ell_{q}^{n}\right)} \leqslant \varepsilon n^{(1 / q-1 / s)_{+}}\right\} \\
\geqslant \min \left\{\|E-U\|_{B\left(\ell_{p}^{n}, \ell_{q}^{n}\right)}: U \in \mathscr{L}_{n},\|U\|_{B\left(\ell_{1}^{n}, \ell_{\infty}^{n}\right)} \leqslant \varepsilon\right\} .
\end{array}
$$

Далее, учитывая, что

$$
\begin{aligned}
\|E-U\|_{B\left(\ell_{\infty}^{n}, \ell_{1}^{n}\right)} & \leqslant\|E\|_{B\left(\ell_{q}^{n}, \ell_{1}^{n}\right)}\|E-U\|_{B\left(\ell_{p}^{n}, \ell_{q}^{n}\right)}\|E\|_{B\left(\ell_{\infty}^{n}, \ell_{p}^{n}\right)} \\
& =n^{1-1 / q}\|E-U\|_{B\left(\ell_{p}^{n}, \ell_{q}^{n}\right) n^{1 / p}}
\end{aligned}
$$


или, что то же самое,

$$
\|E-U\|_{B\left(\ell_{p}^{n}, \ell_{q}^{n}\right)} \geqslant n^{1 / q-1 / p-1}\|E-U\|_{B\left(\ell_{\infty}^{n}, \ell_{1}^{n}\right)},
$$

из (2.1.11) и (2.1.5) получаем

$$
\begin{aligned}
\min & \left\{\|E-U\|_{B\left(\ell_{p}^{n}, \ell_{q}^{n}\right)}: U \in \mathscr{L}_{n},\|U\|_{B\left(\ell_{s}^{n}, \ell_{q}^{n}\right)} \leqslant \varepsilon n^{(1 / q-1 / s)+}\right\} \\
& \geqslant n^{1 / q-1 / p-1} \min \left\{\|E-U\|_{B\left(\ell_{\infty}^{n}, \ell_{1}^{n}\right)}: U \in \mathscr{L}_{n},\|U\|_{B\left(\ell_{1}^{n}, \ell_{\infty}^{n}\right)} \leqslant \varepsilon\right\} \\
& =(1-\varepsilon) n^{1 / q-1 / p},
\end{aligned}
$$

что эквивалентно (2.1.10).

4) $p<q<s$.

Учитывая, что в рассматриваемом случае для каждого оператора $U \in \mathscr{L}_{n}$, подчиненного условию (2.1.9), выполняется неравенство

$$
\begin{aligned}
\|U\|_{B\left(\ell_{\infty}^{n}, \ell_{1}^{n}\right)} \leqslant & \|E\|_{B\left(\ell_{q}^{n}, \ell_{1}^{n}\right)}:\|U\|_{B\left(\ell_{s}^{n}, \ell_{q}^{n}\right)}\|E\|_{B\left(\ell_{\infty}^{n}, \ell_{s}^{n}\right)} \\
& =n^{1-1 / q}\|U\|_{B\left(\ell_{s}^{n}, \ell_{q}^{n}\right)} n^{1 / s} \leqslant n^{1-1 / q} \varepsilon n^{1 / q-1 / s} n^{1 / s}=\varepsilon n
\end{aligned}
$$

приходим к выводу, что

$$
\begin{aligned}
& \min \left\{\|E-U\|_{B\left(\ell_{p}^{n}, \ell_{q}^{n}\right)}: U \in \mathscr{L}_{n},\|U\|_{B\left(\ell_{s}^{n}, \ell_{q}^{n}\right)} \leqslant \varepsilon n^{(1 / q-1 / s)_{+}}\right\} \\
& \geqslant \min \left\{\|E-U\|_{B\left(\ell_{p}^{n}, \ell_{q}^{n}\right)}: U \in \mathscr{L}_{n},\|U\|_{B\left(\ell_{\infty}^{n}, \ell_{1}^{n}\right)} \leqslant \varepsilon n\right\} .
\end{aligned}
$$

Ввиду того, что

$$
\|E-U\|_{B\left(\ell_{1}^{n}, \ell_{\infty}^{n}\right)} \leqslant\|E\|_{B\left(\ell_{q}^{n}, \ell_{\infty}^{n}\right)}\|E-U\|_{B\left(\ell_{p}^{n}, \ell_{q}^{n}\right)}\|E\|_{B\left(\ell_{1}^{n}, \ell_{p}^{n}\right)}=\|E-U\|_{B\left(\ell_{p}^{n}, \ell_{q}^{n}\right)}
$$

из (2.1.12), пользуясь (2.1.6), вьводим оценку

$$
\begin{aligned}
& \min \left\{\|E-U\|_{B\left(\ell_{p}^{n}, \ell_{q}^{n}\right)}: U \in \mathscr{L}_{n},\|U\|_{B\left(\ell_{s}^{n}, \ell_{q}^{n}\right)} \leqslant \varepsilon n^{(1 / q-1 / s)+}\right\} \\
& \geqslant \min \left\{\|E-U\|_{B\left(\ell_{1}^{n}, \ell_{\infty}^{n}\right)}: U \in \mathscr{L}_{n},\|U\|_{B\left(\ell_{\infty}^{n}, \ell_{1}^{n}\right)} \leqslant \varepsilon n\right\}=1-\varepsilon
\end{aligned}
$$

которая совпадает с (2.1.10).

Итак, неравенство (2.1.10) установлено для любого оператора $U \in \mathscr{L}_{n}$, подчиненного условию (2.1.9). Сопоставляя сказанное с тем, что для оператора $\varepsilon E \in \mathscr{L}_{n}$ имеют место равенства

$$
\begin{aligned}
\|\varepsilon E\|_{B\left(\ell_{s}^{n}, \ell_{q}^{n}\right)} & =\varepsilon n^{(1 / q-1 / s)_{+},} \\
\|E-\varepsilon E\|_{B\left(\ell_{p}^{n}, \ell_{q}^{n}\right)} & =(1-\varepsilon) n^{(1 / q-1 / p)_{+},}
\end{aligned}
$$

убеждаемся в справедливости (2.1.8).

2.2. В этом пункте будет получен основной результат $\S 2$. Для этого нам понадобятся леммы 2.2.1 и 2.2.2. 
Лемма 2.2.1. Пусть $d \in \mathbb{N}$ и функиия $\varphi \in C^{\infty}\left(\mathbb{R}^{d}\right)$ такова, ито $\varphi \neq 0$ и ее носитель $\operatorname{supp} \varphi \subset I^{d}$. Пусть еще для $m \in \mathbb{N}$ определены $\mathscr{S}_{m}=\mathscr{S}_{m}^{d, \varphi}$ - линейная оболочка системы функиий $\left\{\varphi(m x-\nu), \nu \in \mathrm{N}_{m}^{d}\right\}$ и $J_{m}=J_{m}^{d, \varphi}: \mathbb{R}^{m^{d}} \rightarrow \mathscr{S}_{m}^{d, \varphi}$ - линейный оператор, задаваемый равенством

$$
J_{m} \beta=\sum_{\nu \in \mathrm{N}_{m}^{d}} \beta_{\nu} \varphi(m x-\nu), \quad \beta=\left\{\beta_{\nu} \in \mathbb{R}, \nu \in \mathrm{N}_{m}^{d}\right\} .
$$

Тогда

1) для любого $m \in \mathbb{N}$ система функиий $\left\{\varphi(m x-\nu), \nu \in \mathrm{N}_{m}^{d}\right\}$ линейно независима, и $J_{m}$ суть линейный изоморфизм $\mathbb{R}^{m^{d}}$ на $\mathscr{S}_{m} ;$

2) для любого $r: 1 \leqslant r \leqslant \infty$ существует константа $c_{8}(r, d, \varphi)>0$ такая, что для любих $m \in \mathbb{N}, \beta \in \mathbb{R}^{m^{d}}$ справедливо равенство

$$
\left\|J_{m} \beta\right\|_{L_{r}\left(I^{d}\right)}=c_{8} m^{-d / r}\|\beta\|_{\ell_{r}^{m}}
$$

3) для любых $l \in \mathbb{Z}_{+}, 1 \leqslant p \leqslant \infty$, существует константа $c_{9}(l, p, d, \varphi)>0$ такая, ито при любом модуле непрерьвности $\omega$ илюбом $m \in \mathbb{N}$ имеет место включение

$$
J_{m}\left(\left\{\beta \in \mathbb{R}^{m^{d}}:\|\beta\|_{\ell_{p}^{m}} \leqslant c_{9} m^{-l+d / p} \omega\left(m^{-1}\right)\right\}\right) \subset F_{p}^{l, \omega}\left(I^{d}\right) .
$$

ДокаЗАТЕЛЬСтво. Первое утверждение леммы, очевидно,верно.

Чтобы проверить справедливость второго, заметим, что для $\lambda \in \mathbb{Z}_{+}^{d}, 1 \leqslant r<\infty$, имеет место равенство

$$
\begin{aligned}
\left\|\mathscr{D}^{\lambda} J_{m} \beta\right\|_{L_{r}\left(I^{d}\right)} & =\left(\int_{I^{d}}\left|\sum_{\nu \in \mathrm{N}_{m}^{d}} \beta_{\nu} \mathscr{D}^{\lambda}(\varphi(m x-\nu))\right|^{\nu} d x\right)^{1 / \nu} \\
& =\left(\sum_{\mu \in \mathrm{N}_{m}^{d}} \int_{m^{-1}\left(\mu+I^{d}\right)}\left|\sum_{\nu \in \mathrm{N}_{m}^{d}} \beta_{\nu} m^{|\lambda|} \mathscr{D}^{\lambda} \varphi(m x-\nu)\right|^{\nu} d x\right)^{1 / \nu} \\
& =m^{|\lambda|}\left(\sum_{\nu \in \mathrm{N}_{m}^{d}} \int_{m^{-1}\left(\nu+I^{d}\right)}\left|\beta_{\nu} \mathscr{D}^{\lambda} \varphi(m x-\nu)\right|^{\nu} d x\right)^{1 / \nu} \\
& =m^{|\lambda|}\left(\sum_{\nu \in \mathrm{N}_{m}^{d}}\left|\beta_{\nu}\right|^{\nu} \int_{I^{d}}\left|\mathscr{D}^{\lambda} \varphi(y)\right|^{\nu} m^{-d} d y\right)^{1 / \nu} \\
& =c_{10}(r, \lambda, d, \varphi) m^{|\lambda|-d / r}\|\beta\|_{\ell_{r}^{m d}}, \beta=\left\{\beta_{\nu}, \nu \in \mathrm{N}_{m}^{d}\right\} \in \mathbb{R}^{m^{d}} .
\end{aligned}
$$

Ясно, что равенство $\left(2.2 .1^{\prime}\right)$ сохраняет свою силу и при $r=\infty$. Полагая в $\left(2.2 .1^{\prime}\right)$ $\lambda=0$, получаем (2.2.1).

Теперь докажем третье утверждение леммы.

Пусть для $\beta=\left\{\beta_{\nu}, \nu \in \mathrm{N}_{m}^{d}\right\}$ соблюдается неравенство

$$
\|\beta\|_{\ell_{p}^{m^{d}}} \leqslant c_{9} m^{-l+d / p} \omega\left(m^{-1}\right)
$$


с константой $c_{9}(l, p, d, \varphi)$, которую мы выберем ниже. Тогда для $g=J_{m} \beta$ при $\lambda \in \mathbb{Z}_{+}^{d}$ в силу $\left(2.2 .1^{\prime}\right)$ имеем

$$
\left\|\mathscr{D}^{\lambda} g\right\|_{L_{p}\left(I^{d}\right)} \leqslant c_{10} c_{9} m^{-l+|\lambda|} \omega\left(m^{-1}\right) .
$$

Пользуясь (2.2.3), для $\varkappa \in \mathbb{Z}_{+l}^{\prime d}$ получаем

$$
\begin{aligned}
\left\|\mathscr{D}^{\varkappa} g(x+h)-\mathscr{D}^{\varkappa} g(x)\right\|_{L_{p}\left(I_{h}^{d}\right)} & \leqslant\left\|\mathscr{D}^{\varkappa} g(x+h)\right\|_{L_{p}\left(I_{h}^{d}\right)}+\left\|\mathscr{D}^{\varkappa} g\right\|_{L_{p}\left(I_{h}^{d}\right)} \\
& \leqslant 2\left\|\mathscr{D}^{\varkappa} g\right\|_{L_{p}\left(I^{d}\right)} \leqslant 2 c_{10} c_{g} \omega\left(m^{-1}\right) \\
& \leqslant 2 c_{10} c_{g} \omega(\|h\|)
\end{aligned}
$$

при $h \in \mathbb{R}^{d},\|h\| \geqslant m^{-1}$,

$$
\begin{aligned}
\left\|\mathscr{D}^{\varkappa} g(x+h)-\mathscr{D}^{\varkappa} g(x)\right\|_{L_{p}\left(I_{h}^{d}\right)} & =\left\|\int_{0}^{1} \sum_{j=1}^{d} \mathscr{D}^{\varkappa+\varepsilon_{j}} g(x+t h) h_{j} d t\right\|_{L_{p}\left(I_{h}^{d}\right)} \\
& \leqslant \int_{0}^{1}\left\|\sum_{j=1}^{d} \mathscr{D}^{\varkappa+\varepsilon_{j}} g(x+t h) h_{j}\right\|_{L_{p}\left(I_{h}^{d}\right)} d t \\
& \leqslant \int_{0}^{1} \sum_{j=1}^{d}\left|h_{j}\right|\left\|\mathscr{D}^{\varkappa+\varepsilon_{j}} g\right\|_{L_{p}\left(I_{h}^{d}\right)} d t \\
& \leqslant \sum_{j=1}^{d}\left|h_{j}\right| c_{9} c_{10}\left(\varkappa+\varepsilon_{j}, \cdot\right) m \omega\left(m^{-1}\right) \\
& \leqslant c_{9} m \omega\left(m^{-1}\right)\|h\| \sum_{j=1}^{d} c_{10}\left(\varkappa+\varepsilon_{j}, \cdot\right) \\
& \leqslant 2 c_{9} \omega(\|h\|) \sum_{j=1}^{d} c_{10}\left(\varkappa+\varepsilon_{j}, \cdot\right)
\end{aligned}
$$

при $h \in \mathbb{R}^{d},\|h\| \leqslant m^{-1}$.

Отсюда видно, что можно выбрать $c_{9}(l, p, d, \varphi)>0$ так, чтобы имело место включение (2.2.2).

Лемма 2.2.2. Пусть $d \in \mathbb{N}$ и функиия $\varphi \in C^{\infty}\left(\mathbb{R}^{d}\right)$ такова, ито $\varphi \neq 0$ и ее носитель $\operatorname{supp} \varphi \subset I^{d}$. Тогда при любом $\lambda \in \mathbb{Z}_{+}^{d}$ существуют семейство констант $\left\{c_{11}(q, \lambda, d, \varphi)>0,1 \leqslant q \leqslant \infty\right\}$ и последовательность линейных операторов

$$
\pi_{m}=\pi_{m}^{\lambda, d, \varphi}: L_{1}\left(I^{d}\right) \rightarrow \mathscr{S}_{m}^{d, \varphi}, \quad m \in \mathbb{N}
$$

(см. лемму 2.2.1), такие, что

1) при $m \in \mathbb{N}$ для $f \in \mathscr{S}_{m}$ справедливо равенство

$$
\pi_{m} \mathscr{D}^{\lambda} f=m^{|\lambda|} f
$$

2) при $m \in \mathbb{N}$ для $f \in L_{q}\left(I^{d}\right), 1 \leqslant q \leqslant \infty$, выполняется неравенство

$$
\left\|\pi_{m} f\right\|_{L_{q}\left(I^{d}\right)} \leqslant c_{11}\|f\|_{L_{q}\left(I^{d}\right)} .
$$


ДокАЗАТЕЛЬСТво. Легко понять, что для функции $\varphi \in C^{\infty}\left(\mathbb{R}^{d}\right)$ такой, что $\varphi \neq 0$ и $\operatorname{supp} \varphi \subset I^{d}$, при $\lambda \in \mathbb{Z}_{+}^{d}$ функция $\mathscr{D}^{\lambda} \varphi \neq 0$ и ее носитель $\operatorname{supp} \mathscr{D}^{\lambda} \varphi \subset I^{d}$. Отсюда ясно, что существует константа $c=c(d, \varphi, \lambda)>0$ такая, что для функции $\psi=c \mathscr{D}^{\lambda} \varphi$ соблюдается условие

$$
\int_{I^{d}} \mathscr{D}^{\lambda} \varphi(x) \psi(x) d x=1
$$

Определим тогда для $m \in \mathbb{N}$ линейный оператор

$$
\pi_{m}=\pi_{m}^{\lambda, d, \varphi}: L_{1}\left(I^{d}\right) \rightarrow \mathscr{S}_{m}^{d, \varphi}
$$

полагая для $f \in L_{1}\left(I^{d}\right)$ значение

$$
\left(\pi_{m} f\right)(x)=m^{d} \sum_{\nu \in \mathrm{N}_{m}^{d}}\left(\int_{I^{d}} f(y) \psi(m y-\nu) d y\right) \varphi(m x-\nu)
$$

Чтобы убедиться в справедливости равенства (2.2.4) для $f \in \mathscr{S}_{m}$, его достаточно проверить для $f=\varphi(m x-\mu)$ при $\mu \in \mathrm{N}_{m}^{d}$. А учитывая, что

$$
\operatorname{supp} \mathscr{D}^{\lambda} \varphi(m x-\mu) \subset m^{-1}\left(\mu+I^{d}\right), \quad \operatorname{supp} \psi(m x-\nu) \subset m^{-1}\left(\nu+I^{d}\right)
$$

и

$$
m^{-1}\left(\mu+I^{d}\right) \cap m^{-1}\left(\nu+I^{d}\right)=\varnothing
$$

при $\mu \neq \nu$, пользуясь $(2.2 .6)$, имеем для $\mu \in \mathrm{N}_{m}^{d}$ :

$$
\begin{aligned}
& \left(\left(\pi_{m} \mathscr{D}^{\lambda}\right)(\varphi(m y-\mu))\right)(x) \\
& \quad=m^{d} \sum_{\nu \in \mathrm{N}_{m}^{d}}\left(\int_{I^{d}} \mathscr{D}^{\lambda}(\varphi(m y-\mu)) \psi(m y-\nu) d y\right) \varphi(m x-\nu) \\
& \quad=m^{d} \sum_{\nu \in \mathrm{N}_{m}^{d}}\left(\int_{I^{d}} m^{|\lambda|} \mathscr{D}^{\lambda} \varphi(m y-\mu) \psi(m y-\nu) d y\right) \varphi(m x-\nu) \\
& \quad=m^{d} m^{|\lambda|}\left(\int_{m^{-1}\left(\mu+I^{d}\right)} \mathscr{D}^{\lambda} \varphi(m y-\mu) \psi(m y-\mu) d y\right) \varphi(m x-\mu) \\
& =m^{|\lambda|}\left(\int_{I^{d}} \mathscr{D}^{\lambda} \varphi(z) \psi(z) d z\right) \varphi(m x-\mu)=m^{|\lambda|} \varphi(m x-\mu) .
\end{aligned}
$$


Проверяя (2.2.5) для $f \in L_{q}\left(I^{d}\right)$ при $1 \leqslant q<\infty$, получаем

$$
\begin{aligned}
& \left\|\pi_{m} f\right\|_{L_{q}\left(I^{d}\right)}^{q}=m^{d q} \int_{I^{d}}\left|\sum_{\nu \in \mathrm{N}_{m}^{d}}\left(\int_{I^{d}} f(y) \psi(m y-\nu) d y\right) \varphi(m x-\nu)\right|^{q} d x \\
& =m^{d q} \sum_{\mu \in \mathrm{N}_{m}^{d}} \int_{m^{-1}\left(\mu+I^{d}\right)}\left|\sum_{\nu \in \mathrm{N}_{m}^{d}}\left(\int_{I^{d}} f(y) \psi(m y-\nu) d y\right) \varphi(m x-\nu)\right|^{q} d x \\
& =m^{d q} \sum_{\mu \in \mathrm{N}_{m}^{d}}\left(\int_{m^{-1}\left(\mu+I^{d}\right)}|\varphi(m x-\mu)|^{q} d x\right)\left|\int_{I^{d}} f(y) \psi(m y-\mu) d y\right|^{q} \\
& =m^{d q-d}\left(\int_{I^{d}}|\varphi(z)|^{q} d z\right) \sum_{\mu \in \mathrm{N}_{m}^{d}}\left|\int_{m^{-1}\left(\mu+I^{d}\right)} f(y) \psi(m y-\mu) d y\right|^{q} \\
& =m^{d q-d}\left(\int_{I^{d}}|\varphi(z)|^{q} d z\right) \\
& \times \sum_{\mu \in \mathrm{N}_{m}^{d}}\left(\int_{m^{-1}\left(\mu+I^{d}\right)}|f(y)|^{q} d y\right)\left(\int_{m^{-1}\left(\mu+I^{d}\right)}|\psi(m y-\mu)|^{q^{\prime}} d y\right)^{q-1} \\
& =m^{d q-d}\left(\int_{I^{d}}|\varphi(z)|^{q} d z\right) \\
& \times m^{-d(q-1)}\left(\int_{I^{d}}|\psi(z)|^{q^{\prime}} d z\right)^{q-1} \sum_{\mu \in \mathrm{N}_{m}^{d}} \int_{m^{-1}\left(\mu+I^{d}\right)}|f(y)|^{q} d y \\
& =\left(\|\varphi\|_{L_{q}\left(I^{d}\right)}\|\psi\|_{L_{q^{\prime}}\left(I^{d}\right)}\right)^{q} \int_{I^{d}}|f(y)|^{q} d y \\
& =\left(\|\varphi\|_{L_{q}\left(I^{d}\right)}\|\psi\|_{L_{q^{\prime}}\left(I^{d}\right)}\|f\|_{L_{q}\left(I^{d}\right)}\right)^{q}, \quad q^{\prime}=\frac{q}{q-1},
\end{aligned}
$$

что влечет (2.2.5).

Аналогично проверяется (2.2.5) при $q=\infty$.

С помощью теоремы 2.1.6, лемм 2.2.1 и 2.2.2 доказывается

ТЕОРЕМА 2.2.3. В условиях теоремы 1.2 .1 существуют константы $c_{12}(d, q, s, \lambda)>0$ u $c_{13}(l, d, p, q, \lambda)>0$ такие, что для любого $m \in \mathbb{N}$ вйполняется неравенство

$$
\mathbf{E}\left(A, X, Y, K, c_{12} m^{\theta}\right) \geqslant c_{13} m^{-\gamma} \omega\left(m^{-1}\right) .
$$

ДокАЗАТЕЛьСтво. Фиксировав функцию $\varphi \in C^{\infty}\left(\mathbb{R}^{d}\right)$, носитель которой $\operatorname{supp} \varphi \subset I^{d}$, и такую, что $\varphi \neq 0$, построим последовательности подпространств $\mathscr{S}_{m}^{d, \varphi}$ и операторов $J_{m}^{d, \varphi}$ и $\pi_{m}^{\lambda, d, \varphi}, m \in \mathbb{N}$, указанные в леммах 2.2 .1 и 2.2.2.

Тогда ввиду (2.2.4) для $m \in \mathbb{N}, \beta=\left\{\beta_{\nu}, \nu \in \mathrm{N}_{m}^{d}\right\}$ имеем

$$
m^{|\lambda|} \beta=m^{|\lambda|}\left(J_{m}\right)^{-1} J_{m} \beta=\left(J_{m}\right)^{-1} m^{|\lambda|} J_{m} \beta=\left(J_{m}\right)^{-1} \pi_{m} \mathscr{D}^{\lambda} J_{m} \beta
$$


Отсюда в силу (2.2.1) и (2.2.5) для $V \in B\left(L_{s}\left(I^{d}\right), L_{q}\left(I^{d}\right)\right)$ при $m \in \mathbb{N}$ для $\beta \in \mathbb{R}^{m^{d}}$ получаем оценку

$$
\begin{aligned}
\left\|m^{|\lambda|} \beta-\left(J_{m}\right)^{-1} \pi_{m} V J_{m} \beta\right\|_{\ell_{q}^{m^{d}}} & =\left\|\left(J_{m}\right)^{-1} \pi_{m}\left(\mathscr{D}^{\lambda} J_{m} \beta-V J_{m} \beta\right)\right\|_{\ell_{q}^{m^{d}}} \\
& =\frac{1}{c_{8}} m^{d / q}\left\|\pi_{m}\left(\mathscr{D}^{\lambda} J_{m} \beta-V J_{m} \beta\right)\right\|_{L_{q}\left(I^{d}\right)} \\
& \leqslant \frac{c_{11}}{c_{8}} m^{d / q}\left\|\mathscr{D}^{\lambda} J_{m} \beta-V J_{m} \beta\right\|_{L_{q}\left(I^{d}\right)} .
\end{aligned}
$$

Из условий теоремы, обеспечивающих вложения $F_{p}^{l, \omega}\left(I^{d}\right) \subset L_{s}\left(I^{d}\right)$, $\mathscr{D}^{\lambda}\left(F_{p}^{l, \omega}\left(I^{d}\right)\right) \subset L_{q}\left(I^{d}\right)$, а также из (2.2.2) и (2.2.8) вытекает, что для $V \in$ $B\left(L_{s}\left(I^{d}\right), L_{q}\left(I^{d}\right)\right)$ при $m \in \mathbb{N}$ выполняется неравенство

$$
\begin{aligned}
& \sup \left\{\left\|\mathscr{D}^{\lambda} f-V f\right\|_{L_{q}\left(I^{d}\right)}: f \in F_{p}^{l, \omega}\left(I^{d}\right)\right\} \\
& \geqslant \sup \left\{\left\|\mathscr{D}^{\lambda} J_{m} \beta-V J_{m} \beta\right\|_{L_{q}\left(I^{d}\right)}: \beta \in \mathbb{R}^{m^{d}},\right. \\
& \left.\|\beta\|_{\ell_{p}^{m^{d}}} \leqslant c_{9} m^{-l+d / p} \omega\left(m^{-1}\right)\right\} \\
& \geqslant \sup \left\{\frac{c_{8}}{c_{11}} m^{-d / q}\left\|m^{|\lambda|} \beta-\left(J_{m}\right)^{-1} \pi_{m} V J_{m} \beta\right\|_{\ell_{q}^{m^{d}}}: \beta \in \mathbb{R}^{m^{d}},\right. \\
& \left.\|\beta\|_{\ell_{p}^{m^{d}}} \leqslant c_{9} m^{-l+d / p} \omega\left(m^{-1}\right)\right\} \\
& =\sup \left\{\frac{c_{8}}{c_{11}} m^{-d / q} c_{9} m^{-l+d / p} \omega\left(m^{-1}\right)\right. \\
& \left.\times m^{|\lambda|}\left\|\beta-m^{-|\lambda|}\left(J_{m}\right)^{-1} \pi_{m} V J_{m} \beta\right\|_{\ell_{q}^{m^{d}}}: \beta \in \mathbb{R}^{m^{d}},\|\beta\|_{\ell_{p}^{m^{d}}} \leqslant 1\right\} \\
& \quad \times c_{14} m^{-l+|\lambda|+d / p-d / q} \omega\left(m^{-1}\right) \\
& \quad\left\|E-m^{-|\lambda|}\left(J_{m}\right)^{-1} \pi_{m} V J_{m}\right\|_{B\left(\ell_{p}^{m^{d}}, \ell_{q}^{m^{d}}\right)} .
\end{aligned}
$$

Далее, поскольку для $V \in B\left(L_{s}\left(I^{d}\right), L_{q}\left(I^{d}\right)\right)$ при $m \in \mathbb{N}$ ввиду (2.2.1) и (2.2.5) имеет место оценка

$$
\begin{aligned}
\left\|m^{-|\lambda|}\left(J_{m}\right)^{-1} \pi_{m} V J_{m}\right\|_{B\left(\ell_{s}^{m^{d}}, \ell_{q}^{m^{d}}\right)} & \\
\leqslant & m^{-|\lambda|}\left\|\left(J_{m}\right)^{-1}\right\|_{B\left(\mathscr{S}_{m} \cap L_{q}\left(I^{d}\right), \ell_{q}^{m^{d}}\right)}\left\|\pi_{m}\right\|_{B\left(L_{q}\left(I^{d}\right), L_{q}\left(I^{d}\right)\right)} \\
\quad & \times\|V\|_{B\left(L_{s}\left(I^{d}\right), L_{q}\left(I^{d}\right)\right)}\left\|J_{m}\right\|_{B\left(\ell_{s}^{m^{d}}, L_{s}\left(I^{d}\right)\right)} \\
\leqslant & m^{-|\lambda|} \frac{1}{c_{8}(q, \cdot)} m^{d / q} c_{11}\|V\|_{B\left(L_{s}\left(I^{d}\right), L_{q}\left(I^{d}\right)\right)} c_{8}(s, \cdot) m^{-d / s} \\
= & c_{15} m^{-|\lambda|-d / s+d / q}\|V\|_{B\left(L_{s}\left(I^{d}\right), L_{q}\left(I^{d}\right)\right)},
\end{aligned}
$$

то при $m \in \mathbb{N}$ и $\rho>0$ для $V \in B\left(L_{s}\left(I^{d}\right), L_{q}\left(I^{d}\right)\right),\|V\|_{B\left(L_{s}\left(I^{d}\right), L_{q}\left(I^{d}\right)\right)} \leqslant \rho$, справедливо неравенство

$$
\begin{aligned}
&\left\|E-m^{-|\lambda|}\left(J_{m}\right)^{-1} \pi_{m} V J_{m}\right\|_{B\left(\ell_{p}^{m^{d}}, \ell_{q}^{m^{d}}\right)} \\
& \geqslant \min \left\{\|E-U\|_{B\left(\ell_{p}^{m^{d}}, \ell_{q}^{m^{d}}\right)}: U \in \mathscr{L}_{m^{d}},\right. \\
&\left.\|U\|_{B\left(\ell_{s}^{m^{d}}, \ell_{q}^{m^{d}}\right)} \leqslant c_{15} m^{-|\lambda|-d / s+d / q} \rho\right\} .
\end{aligned}
$$


Объединяя (2.2.9) и (2.2.10), заключаем, что при $m \in \mathbb{N}$ и $\rho>0$ для любого оператора $V \in B\left(L_{s}\left(I^{d}\right), L_{q}\left(I^{d}\right)\right)$, у которого $\|V\|_{B\left(L_{s}\left(I^{d}\right), L_{q}\left(I^{d}\right)\right)} \leqslant \rho$, выполняется неравенство

$$
\begin{aligned}
& \sup _{f \in F_{p}^{l, \omega}\left(I^{d}\right)}\left\|\mathscr{D}^{\lambda} f-V f\right\|_{L_{q}\left(I^{d}\right)} \\
& \geqslant c_{14} m^{-l+|\lambda|+d / p-d / q} \omega\left(m^{-1}\right) \\
& \quad \times \min \left\{\|E-U\|_{B\left(\ell_{p}^{m^{d}}, \ell_{q}^{m^{d}}\right)}: U \in \mathscr{L}_{m^{d}},\|U\|_{B\left(\ell_{s}^{m^{d}}, \ell_{q}^{m^{d}}\right)} \leqslant c_{15} m^{-|\lambda|-d / s+d / q} \rho\right\}
\end{aligned}
$$

и, значит,

$$
\begin{aligned}
& \mathbf{E}(A, X, Y, K, \rho) \\
& \geqslant c_{14} m^{-l+|\lambda|+d / p-d / q} \omega\left(m^{-1}\right) \\
& \times \min \left\{\|E-U\|_{B\left(\ell_{p}^{m^{d}}, \ell_{q}^{m^{d}}\right)}: U \in \mathscr{L}_{m^{d}},\|U\|_{B\left(\ell_{s}^{m^{d}}, \ell_{q}^{m^{d}}\right)} \leqslant c_{15} m^{-|\lambda|-d / s+d / q} \rho\right\} . \\
& \text { Полагая здесь } \rho=c_{12} m^{|\lambda|+(d / s-d / q)_{+}} \text {, где } c_{12}=1 /\left(2 c_{15}\right) \text {, получаем: } \\
& \mathbf{E}\left(A, X, Y, K, c_{12} m^{\theta}\right) \\
& \geqslant c_{14} m^{-l+|\lambda|+d / p-d / q} \omega\left(m^{-1}\right) \\
& \times \min \left\{\|E-U\|_{B\left(\ell_{p}^{m^{d}}, \ell_{q}^{m^{d}}\right)}: U \in \mathscr{L}_{m^{d}},\|U\|_{B\left(\ell_{s}^{m^{d}}, \ell_{q}^{m^{d}}\right)} \leqslant \frac{1}{2} m^{d(1 / q-1 / s)+}\right\} .
\end{aligned}
$$

Подставляя в правую часть последнего неравенства (2.1.8) при $\varepsilon=\frac{1}{2}, n=m^{d}$, приходим к оценке

$$
\begin{aligned}
\mathbf{E}\left(A, X, Y, K, c_{12} m^{\theta}\right) & \geqslant c_{14} m^{-l+|\lambda|+d / p-d / q} \omega\left(m^{-1}\right) \frac{1}{2} m^{d(1 / q-1 / p)_{+}} \\
& =c_{13} m^{-\gamma} \omega\left(m^{-1}\right),
\end{aligned}
$$

которая совпадает с (2.2.7).

Непосредственным следствием теоремы 2.2 .3 является

ТЕОРемА 2.2.4. В условиях теоремы 1.2 .1 существуют константы $\rho_{2}(d, q, s, \lambda)>0$ u $c_{16}(l, d, p, q, s, \lambda)>0$ такие, что при $\rho>\rho_{2}$ соблюдается неравенство

$$
\mathbf{E}(A, X, Y, K, \rho) \geqslant c_{16} \rho^{-\gamma / \theta} \omega\left(\rho^{-1 / \theta}\right) .
$$

ДокАЗАТЕЛЬСТво. Положим $\rho_{2}(d, q, s, \lambda)=c_{12}(d, q, s, \lambda)$ и выберем для $\rho>\rho_{2}$ число $m \in \mathbb{N}, m \geqslant 2$, так, чтобы выполнялось неравенство:

$$
c_{12}(m-1)^{\theta}<\rho \leqslant c_{12} m^{\theta} .
$$

Учитывая, что в силу $(2.2 .12)$

$$
\mathbf{E}(A, X, Y, K, \rho) \geqslant \mathbf{E}\left(A, X, Y, K, c_{12} m^{\theta}\right),
$$

и применяя (2.2.7), получаем:

$$
\mathbf{E}(A, X, Y, K, \rho) \geqslant c_{13} m^{-\gamma} \omega\left(m^{-1}\right) .
$$

Отсюда, пользуясь (2.2.12), легко выводим (2.2.11).

Автор благодарит профессора В. М. Тихомирова за внимание к автору и его работе. 


\section{Список литературы}

1. Стечкин C. Б. Наилучшее приближение линейных операторов // Матем. заметки. 1967. T. 1. № 2. C. 137-148.

2. Арестов B. В. Наилучшее приближение неограниченных операторов ограниченными и родственные задачи // Матем. заметки. 1968. Т. 40. № 2. С. 269-285.

3. Тимошин O.A. О наилучшем приближении дифференцируемых операторов с частными производньпии // Матем. заметки. 1989. Т. 46. № 1. С. 78-87.

4. Тимошин О.А. Порядки наилучшего приближения дифференцируемых операторов с частными производнњми // Матем. заметки. 1990. Т. 48. № 4. С. 115-121.

5. Кудрявиев C.H. Некоторые задачи теории приближений для одного класса функций конечной гладкости // Матем. заметки. 1992. Т. 183. № 2. С. 3-20.

6. Кудряв цев $C$. H. Восстановление функций вместе с их производными по значениям функций в заданном числе точек // Изв. РАН. Сер. матем. 1994. Т. 58. №6. С. 79-104.

Институт высокопроизводителњных

Поступила в редакцию

вычислительных систем РАН

03.05.1995 